\section{Subclavian Steal Syndrome as a Common Denominator of Both Acute Coronary Syndrome and Ipsilateral Transient Ischemic Attack}

Andreas Mitsis, MD; Xun Yuan, MD;

Christoph A. Nienaber, MD, PhD

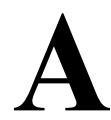
n 82-year-old man was admitted with recurrent angina and transient cerebrovascular ischemic attack (TIA) with ipsilateral (left-sided) hemianopia, associated with temporary dizziness without ischemic lesion on computed tomography. Coronary artery bypass grafting with left internal mammary artery (LIMA) to the left anterior descending artery (LAD) had been performed 18 years previously, and endovascular aneurysm repair 6 years prior to that. Electrocardiography showed sinus rhythm and ST-T depression in the V2-V6 leads, prompting coronary angiography that showed occlusion of the LAD, a patent LIMA and a 70\% left subclavian artery (LSA) stenosis proximal to the LIMA graft. Given that the subclavian stenosis impacted on both the left vertebral artery (perfusing the cerebrum) and LIMA graft (perfusing the anterior myocardium) it was decided to proceed with ad hoc direct stenting of the LSA using an $8-\mathrm{mm} \times 20-\mathrm{mm}$ bare metal stent (Figure A,B). ST-T abnormalities normal- ized immediately after the procedure, allowing the patient to be discharged 2 days later. Twenty-four months after the intervention, the patient remained symptom free with regards to both cerebral and myocardial perfusion. Doppler flow examination confirmed reversal of preinterventional subclavian steal, a phenomenon causing reversed flow from the ipsilateral posterior cerebral circulation and impaired anterior myocardial perfusion via the LIMA. In the present case, stenting of the LSA stenosis had re-established antegrade flow to both the left vertebral artery (posterior cerebral circulation) and to the anterior myocardium via the LIMA (Figure C,D). Appreciation of the pathophysiological complexity and advanced interventional skills are required for proper management in the context of acute coronary syndrome and simultaneous ipsilateral TIA.

\section{Disclosures}

The authors declare no conflicts of interest.

Received May 1, 2018; revised manuscript received June 15, 2018; accepted June 26, 2018; J-STAGE Advance Publication released online August 29, 2018 Time for primary review: 22 days

Cardiology and Aortic Centre, Royal Brompton Hospital and National Heart and Lung Institute of Imperial College London, London, UK

Mailing address: Christoph A. Nienaber, MD, PhD, Cardiology and Aortic Centre, Royal Brompton Hospital and National Heart and Lung Institute of Imperial College London, Sydney Street, London SW3 6NP, UK. E-mail: c.nienaber@rbht.nhs.uk

ISSN-1346-9843 All rights are reserved to the Japanese Circulation Society. For permissions, please e-mail: cj@j-circ.or.jp

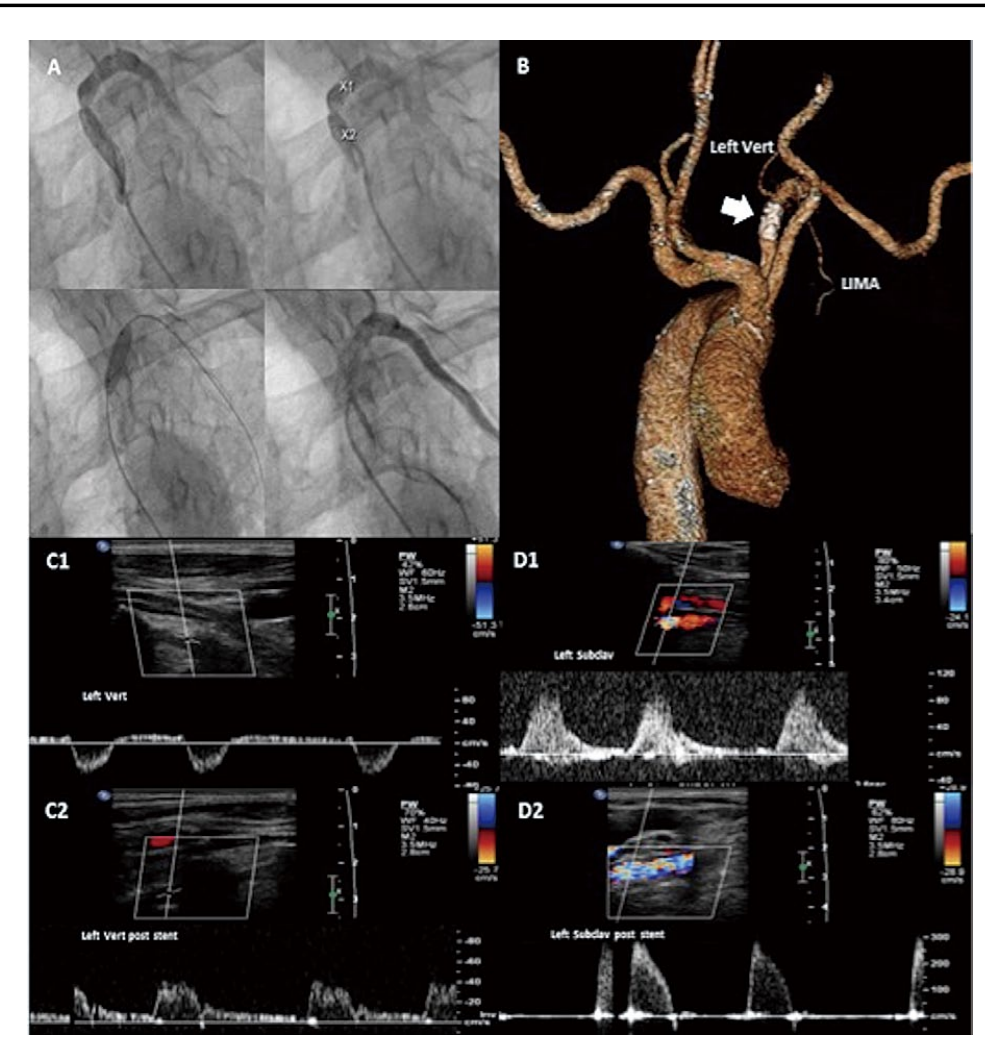

Figure. (A) Angiography showing left subclavian stenosis followed by ad hoc stenting with an 8-mm×20-mm bare metal stent. (B) 3-D reconstructed computed tomography angiogram showing the subclavian stent in close vicinity to the left vertebral artery and left internal mammary artery (LIMA; arrow). (C-1) Doppler flow reversal in the left vertebral artery (before left subclavian artery [LSA] stenting); (C-2) normalized flow signal in left vertebral artery (after LSA stenting). (D-1) Damped flow signal in the LSA (before stenting); (D-2) normalized flow signal in the LSA (after stenting). 